\title{
Experiência e Formação Profissional de Psicoterapeutas Psicanalíticos na Utilização das Tecnologias de Informação e Comunicação ${ }^{1}$
}

\author{
Luan Paris Feijó \\ Universidade do Vale do Rio dos Sinos, RS, Brasil. \\ Nathália Bohn Silva \\ Universidade do Vale do Rio dos Sinos, RS, Brasil. \\ Silvia Pereira da Cruz Benetti \\ Universidade do Vale do Rio dos Sinos, RS, Brasil.
}

\begin{abstract}
Resumo: A crescente utilização das tecnologias de informação e comunicação (TICs) em psicoterapia psicanalítica presencial faz com que seja necessário refletir sobre como os profissionais da área clínica têm percebido o ingresso desses recursos na sua prática e como eles se preparam para manejar com essas tecnologias de forma ética e segura em psicoterapia. Portanto, os objetivos deste estudo foram os de compreender a percepção de psicoterapeutas de orientação psicanalítica sobre a utilização das tecnologias de informação e comunicação com os seus pacientes; identificar o uso das TICs em diferentes níveis de funcionamento psicopatológicos e verificar os conhecimentos científicos dos psicoterapeutas sobre as suas formações profissionais. Foi realizada uma pesquisa qualitativa e exploratória com 11 psicólogos com especialização em psicoterapia psicanalítica. Eles responderam a uma ficha de dados sociodemográficos e à entrevista semiestruturada. Os dados foram analisados por meio de análise temática. Os resultados demonstraram que as tecnologias de informação e comunicação podem servir tanto como uma ferramenta de trabalho como também apresentar entraves à prática profissional. Somado a isso, a falta de instrumentalização pode dificultar a adesão à prática de psicoterapia nesses novos meios tecnológicos. O estudo contribuiu para identificar diferentes formas de se usar as mídias sociais em psicoterapia e auxiliar na ampliação das discussões sobre a temática.
\end{abstract}

Palavras-chave: Psicologia Clínica, Psicoterapia, Psicanálise, Mídias Sociais.

\section{Experience and Professional Training of Psychoanalytic Psychotherapists in the Use of Information and Communication Technologies}

\begin{abstract}
The increasing use of information and communication technologies (ICTs) in face-to-face psychoanalytic psychotherapy makesitnecessary to reflect on how clinical professionals have perceived the inflow of these resources into their practice and how they prepare to handle these technologies in an ethical and safe manner in psychotherapy. In this context, the aim of this study was to understand psychotherapists' perceptions regarding the use of information and communication technologies on their patients, identify the use of ICTs at different psychic functioning levels and verify psychotherapists' scientific knowledge concerning their professional training. Qualitative and exploratory research was carried out with 11 psychologists specialized in psychoanalytic psychotherapy. The psychologists filled out a sociodemographic data sheet and answered a semi-structured interview. Data was analyzed through a thematic analysis. The results indicate that information and communication technologies can serve both as a worktool and as a barrierto professional practice. In addition, lack of instrumentation may hinder psychotherapy practice adherence in these new technological environments. This study contributed to identifying different ways of using social media in psychotherapy and aided in broadening discussions on the subject.
\end{abstract}

Keywords: Psychology, Clinical, Psychotherapy, Psychoanalysis, Social Media.

${ }^{1}$ Artigo oriundo da dissertação de mestrado em Psicologia clínica do primeiro autor deste artigo. 


\title{
Experiencia y Formación Profesional de Psicoterapeutas Psicoanalíticos en la Utilización de las Tecnologías de Información y Comunicación
}

\begin{abstract}
Resumen: La creciente utilización de las tecnologías de información (TIC) y comunicación en psicoterapia psicoanalítica presencial hace que sea necesario reflexionar sobre cómo los profesionales del área clínica han percibido el ingreso de esos recursos en su práctica y cómo ellos se preparan para manejar con esas tecnologías de forma ética y segura en psicoterapia. Por lo tanto, los objetivos de este estudio fueron los de comprender la percepción de psicoterapeutas de orientación psicoanalítica sobre la utilización de las tecnologías de información y comunicación con sus pacientes; identificar el uso de las TIC en diferentes niveles de funcionamiento psicopatológicos y verificar los conocimientos científicos de los psicoterapeutas sobre sus formaciones profesionales. Se realizó una investigación cualitativa y exploratoria con 11 psicólogos con especialización en psicoterapia psicoanalítica. Ellos respondieron a una ficha de datos sociodemográficos y a la entrevista semiestructurada. Los datos se analizaron mediante análisis temáticos. Los resultados demostraron que las tecnologías de la información y la comunicación pueden servir tanto como una herramienta de trabajo, así como para obstaculizar la práctica profesional. Sumado a ello, la falta de instrumentalización puede dificultar la adhesión a la práctica de psicoterapia en esos nuevos medios tecnológicos. El estudio contribuyó a identificar diferentes formas de usar los medios sociales en psicoterapia y auxiliar en la ampliación de las discusiones sobre la temática.
\end{abstract}

Palabras clave: Psicología Clínica, Psicoterapia, Psicoanálisis, Medios de Comunicación Sociales.

\section{Introdução}

As tecnologias de informação e comunicação (TICs) estão presentes na sociedade brasileira (Instituto Brasileiro de Geografia e Estatística [IBGE], 2013). Dentre essas, estão o uso do WhatsApp, Instagram, Facebook e de outras formas tecnológicas. Devido à sua crescente inserção, elas podem influenciar a forma como nos relacionamos com outros indivíduos (Migone, 2013).

Esse impacto não se dá somente no contexto de interação social diário, mas também nos tratamentos psicoterápicos oferecidos (Stadter, 2013). Os trabalhos publicados sobre o tema indicam que as TICs em psicoterapia podem ser usadas como um complemento que auxilia o processo psicoterápico (Distéfano, Mongelo, O'Conor, \& Lamas, 2015) ou como uma ferramenta comunicativa que pode interferir na relação terapêutica e no processo psicoterapêutico (Lisondo, 2012).

Sob esse aspecto, diversos pesquisadores discutem a forma que essas tecnologias influenciam os tratamentos psicoterápicos disponibilizados pelos psicoterapeutas e os benefícios e prejuízos advindos de sua utilização (Barnett, 2011; Feijó, Pessota, Silva, \& Benetti, 2016; Nóbrega, 2015; Pieta, \& Gomes, 2014; Sfoggia et al., 2014). Contudo, há poucos estudos sobre a percepção dos profissionais no que se refere à forma como os pacientes acessam essas tecnologias (Hallberg, \& Lisboa, 2016; Nóbrega, 2015), bem como sobre os sentimentos que são despertados nos terapeutas e como esses profissionais têm se preparado, seja através de estudos teóricos, análise e supervisão, para manejar essa demanda que chega aos consultórios clínicos de psicologia (Carlino, 2011; Migone, 2013).

O estudo empírico mais recente sobre a temática no Brasil foi conduzido por Hallberg e Lisboa (2016) que investigaram a percepção e o uso das tecnologias de informação e comunicação por psicoterapeutas no Rio Grande do Sul. Os resultados encontrados evidenciam que, excetuando o recebimento de solicitações de amizade por meio do Facebook, os psicoterapeutas não se sentem incomodados em se comunicar com os seus pacientes através das TICs. Outro resultado importante dessa mesma pesquisa se refere à preocupação dos psicoterapeutas em estudar sobre a temática. Menos da metade dos participantes preocupavam-se em aprofundar seus conhecimentos sobre o impacto das tecnologias em psicoterapia. Contudo, faz-se necessário que o tema seja pesquisado, uma vez que se torna crescente a inserção das tecnologias no contexto de psicoterapia.

Observa-se que, desde os anos 2000, há pesquisas que investigam o impacto da Internet na vida dos 
sujeitos sob a perspectiva de psicoterapeutas (Leitão, \& Nicolaci-da-Costa, 2005). Nesse estudo nacional, já se discutia a identificação de características subjetivas dos pacientes que usavam a Internet, como o prazer, a onipotência e os excessos de tempo e de exposição vivenciados nesse espaço virtual.

Do mesmo modo, diversos autores discutem os impactos da evolução tecnológica na sociedade, as influências das mudanças contemporâneas no psiquismo dos sujeitos e a manifestação destas transformações em psicoterapia (Kowacs, 2014; Laskoski et al., 2013). Kowacs, (2014) e Nóbrega (2015) também discutem a extensão da hipermodernidade tecnológica na prática psicanalítica, sinalizando a necessidade de que essa teoria possa compreender as novas demandas tecnológicas e interpessoais. Portanto, um eixo de pesquisa pertinente de ser investigado está relacionado às experiências dos psicoterapeutas e a suas formações profissionais para o manejo das tecnologias de informação e comunicação em psicoterapia psicanalítica presencial, uma vez que os pacientes que buscam atendimento psicoterápico, de forma crescente, têm procurado esses serviços através de diversas mídias sociais (Kowacs, 2014; Zilberstein, 2015).

A partir dessas questões, diversos profissionais no exercício da sua prática clínica têm se deparado com situações que alteram a configuração da psicoterapia psicanalítica no seu exercício. Tradicionalmente, nesta abordagem, são mantidos os preceitos da associação livre do paciente, a manutenção da transferência e a neutralidade do terapeuta (Freud, 1912/1996; 1914/1996; 1920/1996; Migone, 2013) em um setting que possa se manter estável (Migone, 2013; Zimerman, 1999).

No entanto, o que se percebe nas publicações sobre a temática se refere a implicações teóricas com intuito de que haja adaptação da técnica para inserção das tecnologias de informação e comunicação, fazendo com que os profissionais precisem (re)pensar o seu exercício profissional para abarcar as TICs, sem transgredir os preceitos do processo psicanalítico. Além disso, essas ferramentas tecnológicas podem auxiliar na diminuição do sofrimento psíquico, desde que sejam utilizadas de forma segura e ética (Carlino, 2011; Migone, 2013; Scharff, 2013). Ainda, Carlino (2011) discute que as TICs podem servir como uma forma de presença comunicativa no período em que psicoterapeuta e paciente estão distantes.

Nesse sentido, a literatura psicanalítica indica ser necessário que os terapeutas possam dispor de formação profissional adequada e de competências profissionais para o manejo das TICs (Carlino, 2011), assim como resguardar a manutenção na sua prática profissional do "tripé psicanalítico": análise pessoal, seminários teóricos e supervisão dos casos clínicos (Saraiva, \& Nunes, 2007). Sob esse viés, Carlino (2011) preconiza que a dupla terapeuta-paciente precisa utilizar uma tecnologia de informação e comunicação em que ambos possuam conhecimento, pois um terapeuta despreparado para a utilização dessas tecnologias poderia gerar um impacto negativo no paciente, assim como na inocorrência da simetria, da associação livre e pôr fim do trabalho elaborativo.

Ainda sob o vértice da psicoterapia psicanalítica e o ingresso das tecnologias eletrônicas em psicoterapia, Stadter (2013) discute que essas tecnologias de informação e comunicação podem ser denominadas de $e$-third no setting psicoterápico. $\mathrm{O} e$-third seria entendido como um objeto eletrônico que poderia influenciar a relação entre a dupla terapeuta e paciente. Esse autor também menciona que o $e$-third é diferente do conceito de terceiro analítico, pois este visa ser conceituado como um espaço intersubjetivo criado pela dupla, diferentemente do e-third, que embora também possa potencializar um espaço de reflexão e evidenciar os estados afetivos do paciente, ele pode promover interações fragmentadas e superficiais. Portanto, assim como discutido nos estudos de Carlino (2011), um profissional que utiliza as tecnologias de forma inexperiente pode fomentar resistências ao tratamento, levando à desistência da psicoterapia por parte do paciente.

Migone (2013) discute as tecnologias de informação e comunicação em psicoterapia psicanalítica utilizando o conceito de psicoterapia com parâmetro, sob referencial da Psicologia do Ego. Para o autor, o ingresso dessas tecnologias na prática clínica poderia estar relacionado a déficits de desenvolvimento do ego, sendo indicado aos pacientes que, devido a essas deficiências, seriam incapazes de aderirem ao modelo fundamentado apenas em interpretações do terapeuta. Assim, esses recursos tecnológicos poderiam ser utilizados como uma forma auxiliar a técnica, ingressando nos atendimentos por tempo determinado, até que o paciente pudesse responder satisfatoriamente à técnica psicoterápica clássica.

Outro ponto relevante sobre a temática se refere ao reconhecimento do trabalho psicoterapêutico a partir das tecnologias de informação e comunicação. Em alguns países, como Estados Unidos e Austrália, a 
prática é reconhecida e aceita e há diversos estudos que discutem como utilizar essas tecnologias a favor da psicoterapia psicanalítica (Carlino, 2011; Crestana, 2015; Scharff, 2012; 2013), já no Brasil a temática parece ser recente e sua discussão ainda incipiente (Hallberg, \& Lisboa, 2016; Pieta, \& Gomes, 2014; Sffogia et al., 2014). Entretanto, o que a literatura tem evidenciado é que a psicoterapia psicanalítica por meio de tecnologias de informação e comunicação pode promover as mesmas mudanças terapêuticas e mantém a técnica psicanalítica semelhante ao tratamento presencial (Scharff, 2012). Assim, investigações que permitam reflexões sobre a incorporação e manejo dessas práticas no dia a dia do profissional psicoterapeuta parecem merecer atenção de pesquisadores.

Portanto, da mesma forma que as tecnologias de informação e comunicação promovem mudanças na subjetividade contemporânea e fomentam uma hipermodernidade tecnológica na sociedade (Kowacz, 2014), elas impactam também na prática clínica (Barcellos et al., 2015; Kowacz, 2014; Luz, 2015) e na formação desses profissionais (Carlino, 2011). Nesse sentido, torna-se necessário investigar como essas relações têm se estabelecido na clínica psicanalítica. Assim, o objetivo deste estudo foi o de compreender qual a percepção de psicoterapeutas de orientação psicanalítica sobre a utilização das tecnologias de informação e comunicação com os seus pacientes; identificar a relação entre o uso das tecnologias de informação e comunicação e a utilização em diferentes níveis de funcionamento psíquico e, por fim, verificar os conhecimentos técnico-científicos dos psicoterapeutas psicodinâmicos sobre as suas formações profissionais no que se refere ao uso dos recursos tecnológicos.

\section{Método}

\section{Delineamento}

Este estudo foi delineado em uma abordagem qualitativa, transversal (Breakwell, Hammond, Five-Schaw, \& Smith, 2010) e exploratória (Gerhardt, \& Silveira, 2009). Com o objetivo de assegurar os procedimentos qualitativos, utilizou-se o Checklist Consolidated criteria for reporting qualitative research (Coreq). Esse instrumento está dividido em três eixos de análise que se referem às características da equipe de pesquisa, ao delineamento metodológico do estudo, assim como à análise e às conclusões apresentadas no estudo (Tong, Sainsbury, \& Craig, 2007).

\section{Participantes}

Participaram desta pesquisa, por meio de amostra por conveniência, 11 psicólogos, sendo dez mulheres e um homem, com especialização em nível de pós-graduação em psicoterapia de orientação psicanalítica. Além disso, destaca-se que os participantes realizaram a formação em diferentes instituições de ensino em psicoterapia da região metropolitana de Porto Alegre no Rio Grande do Sul. Essa estratégia foi adotada pelos autores com o intuito de selecionar uma amostra da mesma abordagem teórica sob diferentes perspectivas de ensino. Ainda, neste estudo, foi utilizado o critério de saturação dos dados para o número final de participantes (Fontanella, Ricas, \& Turato, 2008). Quando as informações emitidas pelos participantes passaram a não apresentar novas manifestações em seu conteúdo, encerraram-se as entrevistas. A Tabela apresenta os dados sociodemográficos dos participantes. Embora haja heterogeneidade na idade dos participantes, não houve conflito para a análise dos dados e dos resultados obtidos.

\section{Instrumentos}

Os instrumentos utilizados para a coleta de dados foram: (1) ficha de dados sociodemográficos, composta por perguntas referentes ao tempo de formação acadêmica (graduação, pós-graduação lato sensu ou stricto sensu) idade, sexo e indicação de quais tecnologias de informação e comunicação utilizava na sua prática clínica e (2) entrevista semiestruturada com questões norteadoras, dividida em quatro blocos. Ambos os instrumentos foram construídos pelos autores.

A versão final da entrevista semiestruturada foi elaborada a partir de um estudo-piloto com seis psicólogos que possuíam especialização em psicoterapia de orientação psicanalítica, diferentes dos participantes do estudo. As perguntas exploratórias estavam direcionadas (a) ao ingresso dos recursos tecnológicos na prática clínica, (b) aos impactos desses recursos na técnica psicanalítica, (c) à formação do profissional e (d) ao manejo de casos clínicos através dos recursos tecnológicos. Os profissionais responderam a uma versão-piloto do questionário com 19 perguntas abertas. Após análise das respostas, organizou-se uma nova versão da entrevista com nove questões abertas distribuídas nas mesmas quatro categorias e acrescidas de tópicos exploratórios. 
Tabela

Apresentação das variáveis sociodemográficos dos participantes $(n=11)$.

\begin{tabular}{lcccc}
\hline Variáveis (em anos) & Mínimo & Máximo & Média & $D P$ \\
\hline Idade dos participantes & 27 & 55 & 34,73 & 7,37 \\
Tempo de conclusão da graduação & 4 & 23 & 10,55 & 5,20 \\
Tempo de conclusão da especialização & 1 & 13 & 6,45 & 3,36 \\
Tempo de experiência clínica & 3 & 23 & 10,18 & 5,31 \\
\hline
\end{tabular}

Nota: Análise realizada por meio de estatísticas descritivas através do software SPSS.

\section{Procedimentos de coleta dos dados}

Os participantes do estudo foram contatados por meio de ligação e e-mail. Após o aceite de participação, foi agendado com o profissional a realização do questionário semiestruturado e da entrevista individualmente em consultório privado. Os participantes assinaram o Termo de Consentimento Livre e Esclarecido. Após esse procedimento, deu-se início à gravação do áudio da entrevista. Primeiro, foi preenchida a ficha de dados sociodemográficos e posteriormente a entrevista semiestruturada. As entrevistas duraram em média 35 minutos.

\section{Procedimentos éticos}

A pesquisa esteve amparada pela Resolução $\mathrm{n}^{0}$ 510/2016 do Conselho Nacional de Saúde, órgão do Ministério da Saúde (Brasil, 2016), bem como foi aprovada pelo Comitê de Ética da Universidade do Vale do Rio dos Sinos-Uninsinos (CAE: 60157816.8.0000.5344), por meio do Parecer n ${ }^{\circ}$ 1.790.149.

\section{Procedimentos de análise dos dados}

Os dados foram analisados manualmente por meio da análise temática (AT). Ela é compreendida como uma análise de temas predominantes em entrevistas, seu aspecto realista tem como propósito operar com a realidade dos participantes envolvidos na pesquisa (Braun, \& Clarke, 2006).

Inicialmente foi transcrito o áudio das entrevistas. Após a transcrição, o material foi lido de forma livre. Na segunda leitura, foram assinalados os tópicos exploratórios, categorizados em unidades temáticas no material transcrito. Já na terceira leitura, as unidades temáticas geraram os temas da pesquisa.
As entrevistas foram analisadas e categorizadas por dois juízes independentes, estes juízes não realizaram as entrevistas com os profissionais. Nos casos em que houve divergência na unidade temática, uma terceira juíza foi convidada a categorizar os materiais. Assim, para a solução dos casos contraditórios foi adotado o consenso entre juízes.

Nesse sentido, as comunicações foram reunidas em temas, sendo: (1) experiência dos profissionais na utilização das tecnologias de informação e comunicação na clínica psicoterápica psicanalítica; (2) impactos das tecnologias de informação e comunicação na técnica psicanalítica e (3) formação do profissional para manejo dos recursos tecnológicos na clínica. Neste artigo, serão apresentados os resultados dos temas: (1) experiência dos profissionais na utilização das tecnologias de informação e comunicação na clínica psicoterápica psicanalítica e (3) a formação do profissional para manejo dos recursos tecnológicos na clínica. O tema (2) impactos das tecnologias de informação e comunicação na técnica psicanalítica foi objeto de discussão em outro artigo elaborado pelos autores (Feijó, Silva, \& Benetti, no prelo).

\section{Resultados}

No que se refere à utilização dos recursos tecnológicos de informação e comunicação, o WhatsApp foi a tecnologia mais utilizada entre os psicoterapeutas $(n=11)$. Além deste, os entrevistados usavam em sua prática clínica outras TICs, como o Facebook $(n=9)$ e o Messenger $(n=3)$. Nenhum dos participantes foi procurado em outros meios tecnológicos de informação e comunicação. A seguir, apresentam-se os resultados. 


\section{Experiência dos profissionais na utilização das TICs na clínica psicoterápica psicanalítica}

Percepção dos profissionais sobre o uso das tecnologias de informação e comunicação

O primeiro tema, (1) experiência dos profissionais na utilização das tecnologias de informação e comunicação na clínica psicoterápica psicanalítica, está dividido em dois subtemas: (a) percepção dos profissionais sobre o uso das tecnologias de informação e comunicação e outro sobre o (b) manejo das tecnologias na relação terapeuta-paciente.

O primeiro subtema, percepção dos profissionais sobre o uso das tecnologias de informação e comunicação, reuniu comunicações relacionadas às características dessas ferramentas como facilitadoras do trabalho clínico, assim como os entraves para o exercício profissional. O Quadro 1 sintetiza os resultados:

Como facilitadores do trabalho clínico, percebeu-se que o WhatsApp foi a principal tecnologia de informação e comunicação utilizada como ferramenta de trabalho profissional. O relato exemplifica a utilização dessa tecnologia entre terapeuta e paciente, "eu tenho usado com [...] duas pacientes específicas, que têm Transtorno de Pânico, então quando elas tão muito angustiadas eu já tenho uma combinação prévia de que elas podem me acessar pelo WhatsApp" (participante 3). Outra forma de utilização refere-se às combinações pontuais entre a dupla, conforme o exemplo a seguir: "Muito mais naquela coisa de marcar: 'Queria marcar contigo' ou desmarcar paciente: 'Ah, preciso desmarcar a consulta hoje. Dá para transferir?' Ou 'Eu preciso de uma sessão extra'” (participante 4). Assim as TICs ampliam as possibilidades de intervenção e compreensão do indivíduo por parte do psicoterapeuta.

Entretanto, a utilização das tecnologias de informação e comunicação também pode gerar alguns entraves ao trabalho clínico, como uma comunicação invasiva "essa paciente eu mudei a visualização [do WhatsApp] porque eu via que quando eu estava online à noite, ou em algum momento, ela vinha querer conversar" (participante 2). Em outra situação com uma terapeuta, "a mãe filmou os surtos da criança [paciente] e me mandou por 'Whats' fora do horário em que eu pudesse estar no consultório" (participante 9). Outro entrave encontrado pelos profissionais seria como uma forma comunicativa ilimitada, "Ela insiste em me mandar mensagens no final de semana. Então, quanto deu [os atendimentos]? Qual foi o valor das consultas esse mês para mandar o cheque?" (participante 5) ou "O WhatsApp me incomoda, porque não tem horário” (participante 9). Além disso, gerar uma forma instantânea de comunicação, como se o terapeuta estivesse que estar presente e disponível integralmente para o paciente: "As pessoas [pacientes] mandam What$s A p p$ e ficam esperando a resposta na hora, só que nem sempre eu posso dar a resposta na hora, mas parece que os pacientes não entendem. Alguns ficam até bravos com isso, parece que não entendem" (participante 4).

Ainda quanto aos entraves ao processo clínico, surgiram nos relatos dos participantes o desconforto com as solicitações de amizades por meio do Facebook. O relato a seguir ilustra a percepção dos

\section{Quadro 1}

Experiência dos profissionais na utilização das tecnologias de informação e comunicação na clínica psicoterápica psicanalítica - Percepção dos profissionais sobre o uso das tecnologias de informação e comunicação.

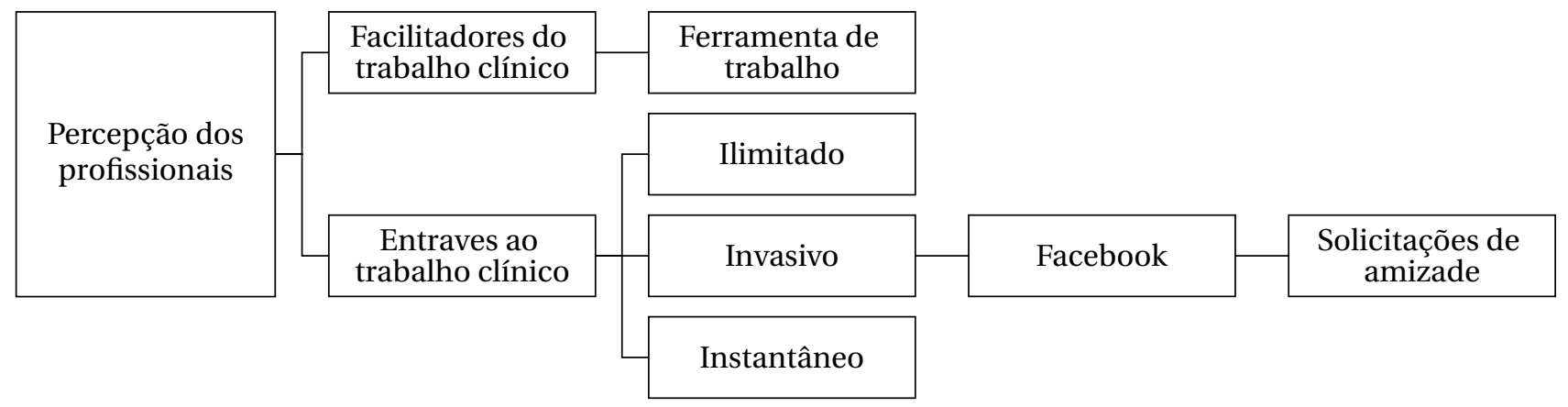

Nota: Quadro elaborado pelos autores. 
psicoterapeutas quanto ao uso do Facebook, "o meu Facebook é bem restrito e eu não aceito convite de ninguém" (participante 3) e "acho que o Facebook é mais pessoal, não acho que os pacientes tenham que me ter nele, não aceito as solicitações porque ali eu posto coisas da minha vida que o paciente não precisa saber" (participante 2). Pode-se perceber que essa mídia social é destinada exclusivamente as informações de caráter pessoal e que as solicitações recebidas dos pacientes são observadas como uma invasão ao seu espaço individual.

\section{Manejo das tecnologias na relação terapeuta-paciente}

O segundo subtema, manejo das tecnologias na relação terapeuta-paciente, reuniu unidades temáticas referentes há como os psicoterapeutas utilizam as tecnologias com os seus pacientes e às intervenções realizadas por meio das tecnologias. O Quadro 2 demonstra os resultados.

Por meio das entrevistas com os profissionais, percebeu-se que eles não realizam contratações prévias sobre o uso das tecnologias com os seus pacientes "eu não faço combinações prévias sobre o uso, eu acho que o paciente vai usar os recursos que ele quiser e a partir disso a gente vai trabalhar" (participante 11). Porém, alguns profissionais defendem que a utilização das tecnologias de informação e comunicação devem estar presentes nas combinações de contrato terapêutico, no início do tratamento "Acho que agora tem que começar a estabelecer novas regras, né. Pra que serve o WhatsApp, né, para não acontecer atuações depois" (participante 4).
Nesse sentido, devido ao uso ser restrito ao contrato terapêutico pelos psicoterapeutas, as intervenções nesses meios têm se diferenciado de acordo com o estilo terapêutico do psicólogo. Enquanto uma pequena parcela dos entrevistados utilizava as TICs independentemente do caso clínico, como com pacientes com um funcionamento psicopatológico grave " $[\ldots]$ um paciente 'border' gravíssimo [...] ele se sentia muito inseguro para a sala de aula. E aí ele me propôs mandar uma mensagem quando ele chegasse na aula e uma mensagem quando ele saísse da aula [...], era uma maneira dele se sentir mais seguro" (participante 10), a maioria dos entrevistados prefere estabelecer a comunicação de forma singular. Ou seja, utilizam de acordo com o vínculo estabelecido e com as necessidades que o terapeuta julga serem necessárias para a relação terapêutica e não com um transtorno psicopatológico, um exemplo dessa escolha está demonstrado na vinheta a seguir "O meu critério é muito a minha contratransferência. Não sei nem se só a psicopatologia, mas acho que aquilo que a pessoa tá vivendo naquele momento" (participante 5).

De modo geral, os psicoterapeutas sinalizam que, com pacientes limítrofes, essas tecnologias deveriam ser utilizadas de forma cautelosa, pois são formas de comunicação passíveis de atuação pelo paciente. Exemplo da restrição evidenciou-se no depoimento a seguir, "eu já fiz [uma combinação] com um paciente 'border'. Combinar de não me mandar tanto 'Whats', da gente combinar que ia me ligar quando não tivesse bem e coisa assim, mas no caso de paciente 'border', eu tive que cortar essa situação porque tava demais” (participante 8).

\section{Quadro 2}

Experiência dos profissionais na utilização das tecnologias de informação e comunicação na clínica psicoterápica psicanalítica - Manejo das tecnologias na relação terapeuta-paciente.

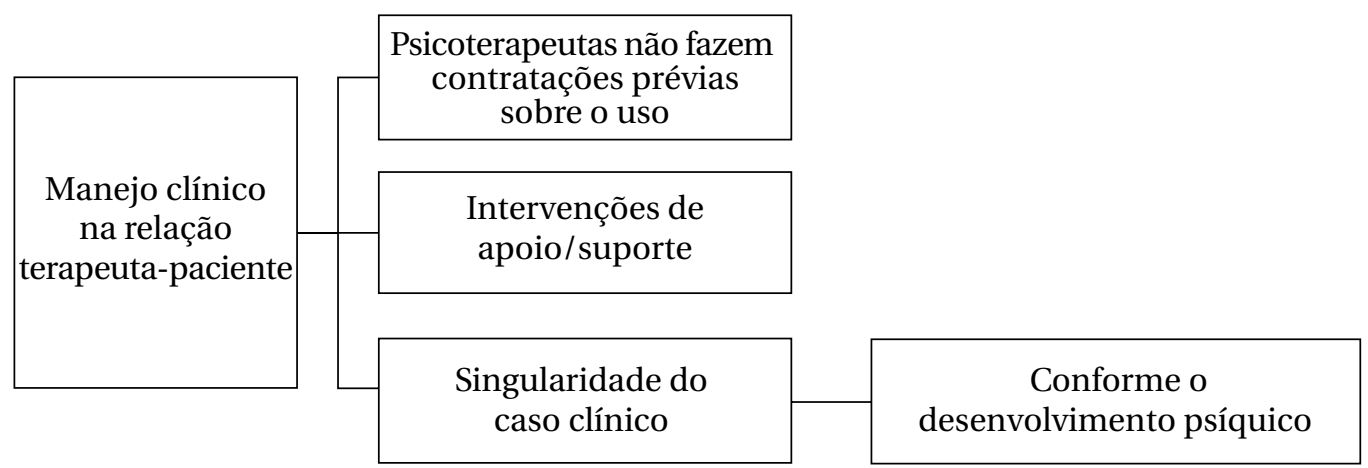

Nota: Quadro elaborado pelos autores 


\section{Formação do profissional para manejo dos recursos tecnológicos na clínica}

O segundo tema, (2) formação do profissional para manejo dos recursos tecnológicos na clínica, encontrou temáticas relacionadas às dificuldades de instrumentalização profissional, bem como à necessidade da inclusão da temática das novas tecnologias de informação e comunicação em psicoterapia no currículo de ensino da Psicologia, tanto em nível de graduação quanto na pós-graduação, e ao estabelecimento de resoluções pelo conselho de classe profissional. O Quadro 3 apresenta as análises realizadas.

Em relação à falta de instrumentalização profissional, os participantes relataram que encontravam pouca literatura sobre o tema "o que eu tenho visto é que saiu na revista, a última do Conselho de Psicologia, acho que foi Federal, mais em relação ao atendimento via Skype, mas eu por enquanto não sei nada sobre mídias, de arranjos ou negociações, eu acho que há uma necessidade de se estudar" (participante 2). Os entrevistados apontaram que seria importante, inclusive, o posicionamento do Conselho de Psicologia no que se refere ao manejo das tecnologias, não apenas em caráter experimental, mas na prática clínica.

Além disso, os profissionais consideraram que não receberam a formação profissional adequada, seja em nível de graduação ou pós-graduação para manejarem as tecnologias no contexto de prática clínica "Não se teve nenhum momento específico pra se falar sobre isso. Se apareceu foi por alguma situação clínica" (participante 1). Em outro exemplo, "a minha formação foi com professoras que não tinham muito acesso a isso" (participante 6). Entretanto, os partici- pantes buscavam a supervisão e os eventos científicos disponíveis na área para aperfeiçoar tecnicamente os seus conhecimentos "acho que se fala pouco sobre isso [tecnologia e psicoterapia] e o espaço em que eu discuto isso às vezes são com colegas ou em supervisão, acho que a gente precisa discutir mais em diferentes espaços acadêmicos" (participante 7). Portanto, essas situações podem evidenciar o despreparo dos profissionais para a utilização e manejo das tecnologias em contexto de psicoterapia.

\section{Discussão}

Por meio das entrevistas, evidenciou-se que a utilização das tecnologias de informação e comunicação no decorrer do exercício profissional dos psicoterapeutas foi presente, pois todos os entrevistados utilizavam o WhatsApp e alguns desses usavam ainda outras formas tecnológicas de informação e comunicação. No entanto, os resultados demonstram que o seu uso pode ser tanto facilitador do trabalho psicodinâmico como também ser uma ferramenta que pode dificultar a prática clínica. Somada a isso, a falta de instrumentalização profissional, assim como as singularidades dos casos clínicos dificultam os manejos profissionais no processo psicoterapêutico.

Em relação à percepção dos profissionais sobre as tecnologias de informação e comunicação como uma ferramenta de trabalho, encontrou-se resultados nesta pesquisa que estão em consonância com o estudo de Hallberg e Lisboa (2016) em que se sugeriu que os psicoterapeutas clínicos tendem a utilizar mais a Internet no contexto profissional do que em outras épocas. Associados às questões éticas e técnicas a

\section{Quadro 3}

Formação do profissional para manejo dos recursos tecnológicos na clínica.

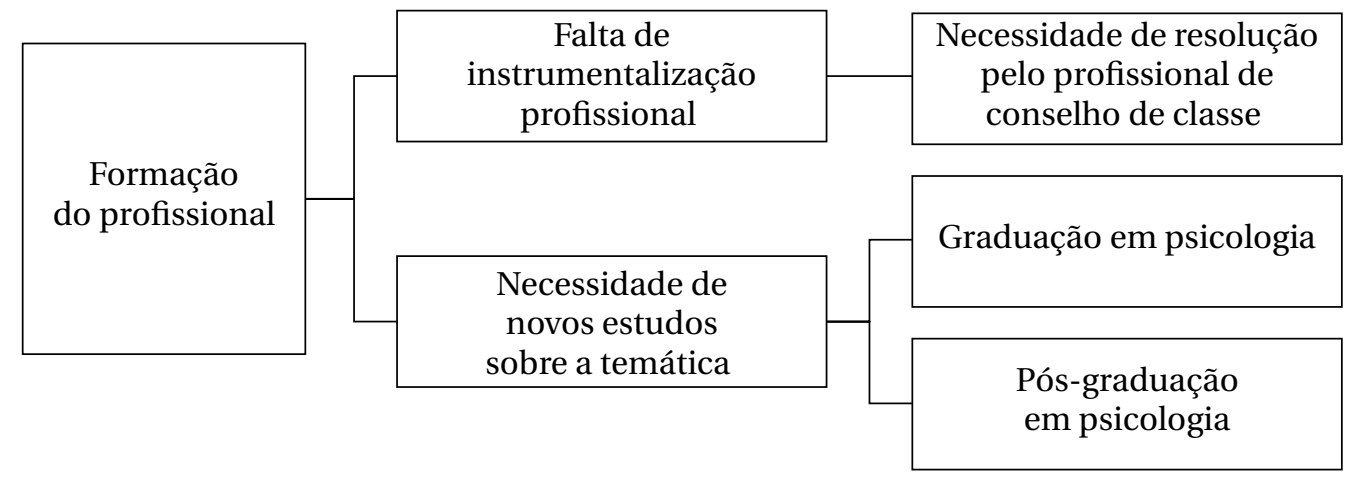

Nota: Quadro elaborado pelos autores. 
psicoterapia, apresenta-se neste artigo um avanço na compreensão desse fenômeno, acrescentando os sentimentos manifestados pelos psicoterapeutas, como ilimitado, invasivo, instantâneo. Ou seja, o fato da baixa adesão às TICs não estaria relacionado somente à falta de informações sobre a eficácia do uso e ao caráter experimental, mas poderia também se relacionar à insegurança dos terapeutas.

Embora diversos estudos contribuam para a discussão dos benefícios nas intervenções psicoterapêuticas por meio das tecnologias de informação e comunicação (Barnett, 2011; Pieta, \& Gomes, 2014; Sfoggia et al., 2014), a percepção dos profissionais participantes da pesquisa é de que mesmo que as TICs sejam consideradas uma ferramenta de trabalho, elas podem proporcionar compreensões negativas sobre seu uso, independentemente da idade do terapeuta participante, pois todos relativizam sobre aspectos positivos e negativos destas ferramentas. Considerando essas questões, pode-se refletir que as situações evidenciadas nos relatos poderiam ser exemplos de manifestações contratransferenciais em decorrência da relação terapêutica, ou seja, as comunicações expressas nessas formas tecnológicas fariam parte de uma presença comunicativa entre a dupla (Carlino, 2011).

O conceito de presença comunicativa é sugerido por Carlino (2011), explicando uma forma de vínculo mesmo quando não há presença física do terapeuta e do paciente em sessão. Nesse caso, há um canal comunicativo entre a dupla, em que estes estão fisicamente longe, mas emocionalmente próximos. Assim, estão passíveis, em conjunto, de estabelecer uma relação de trabalho psicoterapêutico. Esse mesmo autor conclui que não há diferenças significativas entre os diferentes tipos de settings para atendimento, seja presencial ou por tecnologias de informação e comunicação (Carlino, 2011). Nesse sentido, amplia-se a possibilidade de intervenção e manejo nesses novos canais comunicativos. Portanto, o uso das tecnologias pelos pacientes com os seus terapeutas, nesta pesquisa, pode assemelhar ao conceito proposto pelo autor, uma vez que aproxima a dupla, ampliando aspectos psíquicos relacionados ao vínculo da dupla terapêutica.

Ainda, sobre os sentimentos de ilimitado, invasivo, instantâneo pode-se propor que essas manifestações podem ser formas do paciente se aproximar de seu psicoterapeuta em nível mais ou menos integrado, projetando como este sujeito realiza em seus relacionamentos interpessoais. Por exemplo, sob o prisma da
Psicologia Psicanalítica do Ego, um paciente com déficits de ego, que consequentemente pode apresentar prejuízo no seu nível de funcionamento psicopatológico, pode utilizar essas tecnologias justamente para construir condições de estruturar sua capacidade egoica, sendo necessário que o profissional entenda que devido a falhas no desenvolvimento, o paciente utiliza as TICs como uma forma de preencher essas dificuldades (Migone, 2013).

Nesse sentido, Migone (2013), a partir da contribuição de Eissler (1953), discute que o uso dessas tecnologias seria um parâmetro para a técnica psicoterápica, ou seja, um elemento que modifica a combinação de setting estabelecido inicialmente, mas com o intuito de auxiliar temporariamente um paciente. Portanto, parece haver indicações positivas que a teoria psicanalítica possa se atualizar para abarcar de forma ética e coerente essas tecnologias no contexto em que estamos inseridos (Kowacs, 2014; Nóbrega, 2015).

No que se refere ao manejo clínico das tecnologias de informação e comunicação, encontram-se resultados neste estudo demonstrando que os psicoterapeutas usam essas tecnologias em situações pontuais e dependendo de cada sujeito, ou seja, não há uma generalização. O uso clínico é realizado em consideração as singularidades dos sujeitos atendidos pelos terapeutas, indo ao encontro do que se apresenta na literatura sobre o tema (Carlino, 2011; Migone, 2013; Nóbrega, 2015).

Diversos autores acreditam que tanto pacientes com características neuróticas, quanto aqueles com um desenvolvimento psicótico podem se beneficiar da utilização dessas tecnologias, entretanto deve ser avaliado caso a caso (Carlino, 2011; Migone, 2013; Nóbrega, 2015). Os estudos sugerem que poderia ser útil o uso das tecnologias com pacientes adolescentes ansiosos, com aqueles que têm dificuldades no estabelecimento de relacionamentos interpessoais ou contatos mais íntimos com o terapeuta (Carlino, 2011; Migone, 2013). Portanto, os casos apresentados nos relatos deste estudo, um com paciente que apresentava um quadro de ansiedade e outro com um indivíduo identificado com um funcionamento borderline de personalidade, demonstraram que as tecnologias, nessas situações, ingressaram como uma forma de fornecer continência em situações críticas vivenciadas pelos clientes.

Em relação às intervenções por meio das tecnologias de informação e comunicação, foram identificados manejos com caráter de suporte, delineados em 
um modelo de apoio ao paciente, não sendo observadas propostas interpretativas e que gerassem insight por meio das TICs. Possivelmente, por causa das características próprias da psicoterapia psicanalítica, em que se mantém preservado sempre que possível o setting terapêutico para o exercício de questões técnicas e as intervenções psicanalíticas serem menos focais que em outras abordagens (Hallberg, \& Lisboa, 2016), a condução de intervenções expressivas estaria reservada ao processo presencial. Além dessa situação, a falta de instrumentalização profissional, poderia fomentar a não aderência de outras formas de uso pelos psicoterapeutas.

Por fim, a partir das comunicações do eixo formação do profissional para manejo dos recursos tecnológicos na clínica, pode-se refletir que, devido ao exercício psicoterápico online ser uma prática experimental no Brasil (Resoluçao CFP No 011, 2012) e a literatura sobre o tema ainda ser pouco acessível na América Latina, pois a maioria dos estudos estão na língua inglesa (Pires, 2015), os psicoterapeutas acabam por adotar uma postura cautelosa na utilização e no manejo, respeitando os princípios técnicos ensinados nas instituições de formação em relação à psicoterapia psicanalítica (Migone, 2013). Ainda, em muitas delas, como apresentado nos resultados desta pesquisa, não há um espaço para diálogo na especialização para discutir essa temática, ou se possui acaba por ser pouco, reforçando a construção de uma técnica pautada em um saber clássico, por vezes podendo desconsiderar as contribuições contemporâneas na utilização das TICs.

No entanto, se expandir a compreensão da técnica psicoterápica psicanalítica aos países que já adotam a psicoterapia a distância, podemos encontrar estudos sob duas diferentes direções, a primeira sob o prisma que encoraja o uso ético e responsável das tecnologias (Carlino, 2011; Scharff, 2012; 2013) e a segunda sob o ponto de vista daqueles que clamam por cautela e pela adoção da técnica psicanalítica tradicional (Barbieri, 2005; Russell, 2015). Contudo, como relata Nóbrega (2015), a utilização das TICs em psicoterapia já está acontecendo nos consultórios psicoterápicos muito antes dos próprios profissionais discutirem com os seus pares. Portanto, cabe aos pesquisadores e psicoterapeutas clínicos atentarem-se a essa nova forma de comunicação e possibilitarem um diálogo seja na formação, em supervisão ou na sua própria rotina de estudos.

\section{Conclusão}

O presente estudo teve como objetivos compreender a percepção de psicoterapeutas de orientação psicanalítica sobre a utilização das tecnologias de informação e comunicação com os seus pacientes; identificar o uso das TICs em diferentes níveis de funcionamento psicopatológico e, por fim, verificar os conhecimentos científicos dos psicoterapeutas psicodinâmicos sobre as suas formações profissionais. Nesse sentido, os resultados demonstraram que a percepção dos profissionais foi de que os recursos tecnológicos são formas de comunicação que podem facilitar o trabalho terapêutico, servindo como ferramenta de trabalho, mas que também impõem limites no exercício clínico, manifestando compreensões associadas aos sentimentos de invasão, falta de limite e instantaneidade.

Além disso, percebeu-se que os manejos técnicos estavam relacionados com a singularidade do caso clínico e não com níveis de funcionamento psíquicos; portanto, nesses casos, pode ser utilizado tanto com pacientes com graus de funcionamento integrados quanto com pacientes mais primitivos e regressivos. Por fim, a percepção dos profissionais quanto à sua formação foi de que houve a falta de instrumentalização profissional em níveis de graduação e pós-graduação, evidenciando a necessidade de resoluções por parte do conselho profissional.

Cabe considerar que a idade e o tempo de formação profissional não foram objetos de conflito entre as informações apresentadas pelos participantes nas análises realizadas, pois mesmo que se tenha uma amplitude de 28 anos, a formação do psicólogo parece ainda ser bastante restritiva sobre a temática das tecnologias em psicoterapia, pois tem ganhado destaque das pesquisas somente nos últimos anos. Ainda, observou-se que a tecnologia pode ser útil no fazer clínico dos profissionais se estes estiverem bem preparados para o uso das tecnologias de informação e comunicação e disporem de materiais científicos que os auxiliem na formação.

As TICs podem apresentar benefícios aos terapeutas e pacientes, se ambos reconhecerem as possibilidades e limites do uso. Somado a essas questões, as TICs podem servir de parâmetro à técnica psicoterápica preenchendo falhas no desenvolvimento, reatualizadas na vivência com o psicoterapeuta. Contudo, se utilizadas de forma não reflexiva, poderiam gerar abandono ao tratamento, ou atrapalhar o processo em andamento. 
Enquanto direções futuras, sugere-se investigar a percepção dos pacientes sobre o uso dessas tecnologias de informação e comunicação com os seus terapeutas, seja no campo das intervenções presenciais ou a distância. A psicoterapia psicanalítica, conjugada a novas tecnologias de informação e comunicação, desponta como um campo promissor de investigação, pois a tendência parece ser de um crescente uso por parte da população. Desse modo, tanto pesquisadores quanto psicoterapeutas necessitam adotar um modelo reflexivo de estudos para não se prenderem a uma técnica rudimentar e restrita.

\section{Referências}

Barcellos, E. D., Campezatto, P. V. M., Geremia, L., Vieira, M. F., Cavalheiro, R., \& Klarmann, R. P. (2015). As novas formas de comunicação e suas possíveis influências sobre o setting e o campo psicoterápico. Psicoterapia Psicanalítica, 17, 39-51.

Barnett, J. E. (2011). Utilizing technological innovations to enhance psychotherapy supervision, training, and outcomes. Psychotherapy, 48(2), 103-108. https://doi.org/10.1037/a0023381

Barbieri, C. P. (2005). A desregulação da psicanálise. In: Teixeira, A. (Org). Especificidades da ética da psicanálise. Salvador, BA: Associação Científica Campo Psicanalítico.

Brasil. Ministério da Saúde (2016). Conselho Nacional de Saúde. Resolução 510 de 07 de abril de 2016: Dispõe sobre as normas aplicáveis a pesquisas em Ciências Humanas e Sociais. Brasília, DF: Ministério da Saúde.

Braun, V., \& Clarke, V. (2006). Using thematic analysis in psychology. Qualitative Research in Psychology, 3(2), 77-101. https://doi.org/10.1191/1478088706qp063oa

Breakwell, G. M., Hammond, S., Five-Schaw, C. \& Smith, J. A. (2010). Métodos de pesquisa em psicologia (3a ed., F. R. Elizalde, Trad.). Porto Alegre, RS: Artmed.

Carlino, R. (2011). Distance psychoanalysis: the theory and practice of using communication technology in the clinic (J. Nuss, Trad.) Londres: Karnac.

Crestana, T. (2015). Novas abordagens terapêuticas-terapias on-line. Revista Brasileira de Psicoterapia, 17(2), 35-43.

Distéfano, M. J., Mongelo, M. C., O’Conor, J., \& Lamas, M. C. (2015). Psicoterapia y tecnología: implicancias y desafíos en la inserción de recursos innovadores en la práctica clínica argentina. Revista Electrónica de Psicología Iztacala, 18(4): 1342-1362.

Eissler, K. R. (1953). The effect of the structure of the ego on psychoanalytic technique. Journal of the American Psychoanalytic Association, 1(1), 104-143. https://doi.org/10.1177/000306515300100107

Feijó, L. P., Pessota, C. M., Silva, N. B., \& Benetti, S. P. C. (2016). Recursos tecnológicos de comunicação e psicanálise: Revisão sistemática sobre os impactos positivos e negativos na técnica. In Anais do XII Congresso Latino Americano de Pesquisa em Psicoterapia da SPR/LA e IV Simpósio de Pesquisa do Programa de Pós-Graduação em Psicologia da Unisinos. Porto Alegre: Society for Psychotherapy Research.

Feijó, L. P., Silva, N. B., \& Benetti, S. P. C. (no prelo). Impacto das tecnologias de informação e comunicação na técnica psicoterápica psicanalítica. Temas em Psicologia, 26(3).

Fontanella, B. J. B., Ricas, J., \& Turato, E. R. (2008). Amostragem por saturação em pesquisas qualitativas em saúde: contribuições teóricas. Cadernos de Saúde Pública, 24(1), 17-27. https://doi.org/10.1590/S0102$311 X 2008000100003$

Freud. S. (1996). Recomendações aos jovens médicos que exercem a psicanálise. In Edição Standard Brasileira das Obras Psicológicas Completas de Sigmund Freud. Rio de Janeiro, RJ: Imago. (Original publicado em 1912).

Freud, S. (1996). Recordar, repetir e elaborar. In Edição Standard Brasileira das Obras Psicológicas Completas de Sigmund Freud. Rio de Janeiro, RJ: Imago. (Original publicado em 1914).

Freud, S. (1996). Além do princípio do prazer. In Edição Standard Brasileira das Obras Psicológicas Completas de Sigmund Freud. Rio de Janeiro, RJ: Imago. (Original publicado em 1920).

Gerhardt, T. E., \& Silveira, D. T. (2009). Métodos de pesquisa. Porto Alegre, RS: Editora da UFRGS.

Hallberg, S. C. M., \& Lisboa, C. S. M. (2016). Percepção e uso de tecnologias da informação e comunicação por psicoterapeutas. Temas em Psicologia, 24(4), 1297-1309. https://doi.org/10.9788/TP2016.4-06 
Instituto Brasileiro de Geografia e Estatística - IBGE (2013). Pesquisa nacional por amostra de domicílios: Acesso à internet e televisão e posse de telefone móvel celular para uso pessoal. Rio de Janeiro: o autor. Recuperado de http://biblioteca.ibge.gov.br/visualizacao/livros/liv93373.pdf

Kowacs, C. (2014). Prática psicanalítica, tecnologia e hipermodernidade. Revista de Psicanálise da SPPA, 21(3), 629.

Laskoski, P. B., Gastaud, M. B., Goi, J. D., Bassols, A. M. S., Machado, D., ... Eizirik, C. L. (2013). A hipermodernidade e a clínica psicanalítica. Revista Brasileira de Psicoterapia, 15(2), 14-24.

Leitão, C. F., \& Nicolaci-da-Costa, A. M. (2005). Impactos da internet sobre pacientes: a visão de psicoterapeutas. Psicologia em Estudo, 10(3), 441-450. https://doi.org/10.1590/S1413-73722005000300012

Lisondo, A. B. D. (2012). As novas tecnologias que permitem a psicanálise a distância inovam a tradição? Ou elas dificultam a compreensão das novas inovações teóricas e técnicas da psicanálise contemporânea? Montevidéu: Federação Psicanalítica da América Latina.

Luz, A. B. (2015). Oi. Q horas mesmo ficou nossa sessão? TKS. Revista Brasileira de Psicanálise, 49(1), 165-175.

Migone, P. (2013). Psychoanalysis on the Internet: A discussion of its theoretical implications for both online and offline therapeutic technique. Psychoanalytic Psychology, 30(2), 281-299. https://doi.org/10.1037/a0031507

Nóbrega, S. B. (2015). Psicanálise on-line: finalmente saindo do armário?. Estudos de Psicanálise, (44), 145-150..

Pieta, M. A. M., \& Gomes, W. B. (2014). Online therapy: viable or unviable?. Psicologia: Ciência e Profissão, 34(1), 18-31. https://doi.org/10.1590/S1414-98932014000100003

Pires, A. C. J. (2015). Sobre os "tratamentos à distância" em psicoterapia de orientação analítica. Revista Brasileira de Psicoterapia, 17(2), 11-21.

Resolução CFP No 011/ 2012: Regulamenta os serviços psicológicos realizados por meios tecnológicos de comunicação a distância, o atendimento psicoterapêutico em caráter experimental e revoga a Resolução CFP N.o 12/2005. Brasília, DF: Conselho Federal de Psicologia.

Russell, G. I. (2015). Screen relations: The limits of computer-mediated psychoanalysis and psychotherapy. London: Karnac.

Saraiva, L. A., \& Nunes, M. L. T. (2007). A supervisão na formação do analista e do psicoterapeuta psicanalítico. Estudos de Psicologia, 12(3), 259-268. https:// doi.org/10.1590/S1413-294X2007000300008

Scharff, J. S. (2012). Clinical issues in analyses over the telephone and the internet. The International Journal of Psychoanalysis, 93(1), 81-95. https://doi.org/10.1111/j.1745-8315.2011.00548.x.

Scharff, J. S. (2013) Psychoanalysis online: Mental health, teletherapy, and training. London: Karnac.

Sfoggia, A., Kowacs, C., Gastaud, M. B., Laskoski, P. B., Bassols, A. M., .... Eizirik, C. L. (2014). Therapeutic relationship on the web: to face or not to face?. Trends in Psychiatry and Psychotherapy, 36(1), 3-10. https://doi. org/10.1590/2237-6089-2013-0048

Stadter, M. (2013). The influence of social media and communications technology on self and relationships. In J. S. Scharff, Psychoanalysis online: Mental health, teletherapy and training. Londres: Karnac.

Tong, A., Sainsbury, P., \& Craig, J. (2007). Consolidated criteria for reporting qualitative research (COREQ): a 32-item checklist for interviews and focus groups. International Journal for Quality in Health Care, 19(6), 349-357. https:// doi.org/10.1093/intqhe/mzm042

Zilberstein, K. (2015). Technology, relationships and culture: Clinical and theoretical implications. Clinical Social Work Journal, 43(2), 151-158. https://doi.org/10.1007/s10615-013-0461-2

Zimerman, D. E. (1999). Fundamentos psicanalíticos: teoria, técnica, clínica: uma abordagem didática. Porto Alegre,RS: Artmed.

\section{Luan Paris Feijó}

Mestre em Psicologia pela Universidade do Vale do Rio dos Sinos, São Leopoldo - RS. Brasil.

E-mail: lparisf@gmail.com 


\section{Nathália Bohn Silva}

Psicóloga pela Universidade do Vale do Rio dos Sinos, São Leopoldo - RS. Brasil.

E-mail: nathaliabsv@gmail.com

\section{Silvia Pereira da Cruz Benetti}

Professor adjunto no Programa de Pós-Graduação em Psicologia da Universidade do Vale do Rio dos Sinos, São Leopoldo - RS. Brasil.

E-mail: spcbenetti@gmail.com

Endereço para envio de correspondência:

Universidade do Vale do Rio dos Sinos.

Av. Unisinos, 950, Cristo Rei. CEP: 93022-750.

São Leopoldo - RS, Brasil.

Recebido $27 / 07 / 2017$

Aprovado 17/04/2018

Received $07 / 27 / 2017$

Approved 04/17/2018

Recibido 27/07/2017

Aceptado 17/04/2018

Como citar: Feijó, L. P., Silva, N. B., \& Benetti, S. P. C. (2018). Experiência e formação profissional de psicoterapeutas psicanalíticos na utilização das tecnologias de informação e comunicação. Psicologia: Ciência e Profissão, 38(2), 249-261. https://doi.org/10.1590/1982-3703003032017

How to cite: Feijó, L. P., Silva, N. B., \& Benetti, S. P. C. (2018). Experience and professional training of psychoanalytic psychotherapists in the use of information and communication technologies. Psicologia: Ciência e Profissão, 38(2), 249-261. https://doi.org/10.1590/1982-3703003032017

Cómo citar: Feijó, L. P., Silva, N. B., \& Benetti, S. P. C. (2018). Experiencia y formación profesional de psicoterapeutas psicoanalíticos en las tecnologías de información y comunicación. Psicologia: Ciência e Profissão, 38(2), 249-261. https://doi.org/10.1590/1982-3703003032017 